Kragujevac Journal of Mathematics

Volume 45(2) (2021), Pages 181-190.

\title{
A UNITARY TREATMENT OF CERTAIN INEQUALITIES INVOLVING MEANS
}

\author{
A. F. ALBIŞORU ${ }^{1}$ AND M. STROE ${ }^{2}$
}

\begin{abstract}
The aim of this paper is to state and prove certain inequalities that involve means (e.g., the arithmetic, geometric, logarithmic means) using a particular result. First of all we recall useful properties of a real-valued convex function that will be used in the proof of our inequalities. Further, we present three inequalities, the first involving the logarithmic mean, the second involving the classical arithmetical and geometrical means and in the last we introduce a new mean. Finally, we give alternate proofs to the Schweitzer's inequality and Khanin's inequality.
\end{abstract}

\section{INTRODUCTION}

Recall that, for $a, b \in(0, \infty)$, the logarithmic mean is given by the relation

$$
L(a, b):=\frac{b-a}{\log b-\log a},
$$

and for $k \in \mathbb{N} \backslash\{1\}$ and $x_{1}, \ldots, x_{n} \in[a, b]$ we introduce the generalized mean as follows

$$
\sqrt[k]{\frac{x_{1}^{k}+\cdots+x_{n}^{k}}{n}}
$$

These means frequently appear in the setting of inequalities.

The subject of inequalities has fascinated a great deal of mathematicians and the proof of this fact lives in the classical and recent results that bear their names. A large number of inequalities have been the subject of well-known books such as $[1,3,4,6]$.

Note that, an important notion that we employ in this paper is that of a convex function, and we are interesed in the property that the maximum of a convex function

Key words and phrases. Generalized mean, logarithmic mean, convex function, maximum point. 2010 Mathematics Subject Classification. Primary: 26D20. Secondary: 26A51, 26 B25.

DOI 10.46793/KgJMat2102.181A

Received: August 11, 2018.

Accepted: November 05, 2018. 
is attained on the boundary of the convex and bounded domain, on which it is defined (for additional details, see, e.g., $[2,7,8]$ ).

The aim of our work is to establish certain inequalities using convex functions. In Section 2 we give the important notions that will be used throughout the paper. We introduce here the notion of a convex function and we also give a fundamental result which will be used in the proof of our inequalities, namely Theorem 2.1. Through this particular result we point some properties of convex (see, e.g., [5], [7, pp. 89, 118]) functions of several variables and their applications. In Section 3 we have three important applications. Application 3.1 gives an estimate for the difference between the arithmetic and geometric mean in terms of the logarithmic mean. Application 3.2 gives another estimate for the difference between the arithmetic and geometric mean and in Application 3.3 we give an estimate for the difference between the generalized mean and arithmetic mean using a new mean. We end this section with Schweitzer's inequality (Theorem 3.4) and Khanin's Inequality (Theorem 3.5). We have provided alternative proofs for these inequalities using Theorem 2.1.

\section{Prelimiaries}

If $\mathcal{C} \subset \mathbb{R}^{n}$ is a convex set, then, a function $f: \mathcal{C} \rightarrow \mathbb{R}$ is said to be convex if

$$
f((1-\lambda) x+\lambda y) \leq(1-\lambda) f(x)+\lambda f(y), \quad \text { for all } x, y \in \mathcal{C}, \text { for all } \lambda \in[0,1] .
$$

We point out the fact that, if $f:[a, b] \rightarrow \mathbb{R}$ is convex, then the maximum value of $f$ is attained at the boundary. We shall prove this useful property at Lemma 2.1.

In the latter, for $a, b \in \mathbb{R}$, we have the following notations:

$$
\begin{aligned}
{[a, b]^{n} } & =[a, b] \times \cdots \times[a, b], \\
\{a, b\}^{n} & =\{a, b\} \times \cdots \times\{a, b\} .
\end{aligned}
$$

In the latter, we shall prove a theorem that points out that, in certain conditions, the maximum of a convex and continuous function $f:[a, b]^{n} \rightarrow \mathbb{R}$ can be found by taking the maximum of the function on the vertices of the considered hypercube $[a, b]^{n}$, where $a, b \in \mathbb{R}$.

Before we proceed to the proof of this result, we shall state a useful lemma (see, e.g., [7, Theorem 3.4.6, Theorem 3.4.7]).

Lemma 2.1. Let $a, b \in \mathbb{R}$ and let $f:[a, b] \rightarrow \mathbb{R}$ be a convex and continuous function. Then

$$
\max _{x \in[a, b]} f(x)=\max _{x \in\{a, b\}} f(x) .
$$

Proof. Since $f$ is continuous on $[a, b]$, we deduce that there is an $\alpha \in[a, b]$ such that

$$
f(\alpha)=\max _{x \in[a, b]} f(x) .
$$


Now, we argue by contradiction. Assume that $\alpha \in(a, b)$. This means that there is some $\lambda \in(0,1)$, such that

$$
\alpha=(1-\lambda) a+\lambda b
$$

Hence,

$$
\begin{aligned}
f(\alpha) & =f((1-\lambda) a+\lambda b) \leq(1-\lambda) f(a)+\lambda f(b) \\
& <(1-\lambda) f(\alpha)+\lambda f(\alpha),
\end{aligned}
$$

i.e., $f(\alpha)<f(\alpha)$, which is absurd and our proof is complete.

We have the following theorem.

Theorem 2.1. Let $f:[a, b]^{n} \rightarrow \mathbb{R}$ be a $\mathcal{C}^{2}$-class function on $[a, b]^{n}$, such that:

$$
\frac{\partial^{2} f}{\partial x_{i}^{2}} \geq 0, \quad \text { for all } i=\overline{1, n}
$$

Then

$$
\max _{x \in[a, b]^{n}} f(x)=\max _{x \in\{a, b\}^{n}} f(x) .
$$

Proof. Define the function $g_{1}:[a, b] \rightarrow \mathbb{R}$ in the following manner

$$
g_{1}\left(x_{1}\right)=f\left(x_{1}, \ldots, x_{n}\right),
$$

where the variables $x_{2}, \ldots, x_{n}$ are arbitrarily fixed. Using condition (2.1) for $i=1$, we deduce that $g_{1}$ is convex, hence:

$$
\max _{x_{1} \in[a, b]} f\left(x_{1}, \ldots, x_{n}\right)=\max _{x_{1} \in\{a, b\}} f\left(x_{1}, \ldots, x_{n}\right) .
$$

Let $x_{1} \in\{a, b\}$ be the value for which the maximum is attained. We apply the same steps as above to the function $g_{2}:[a, b] \rightarrow \mathbb{R}, g_{2}\left(x_{2}\right)=f\left(x_{1}, x_{2}, \ldots, x_{n}\right)$ and we deduce that there exists $x_{2} \in\{a, b\}$ such that

$$
\max _{x_{2} \in[a, b]} f\left(x_{1}, x_{2}, \ldots, x_{n}\right)=\max _{x_{2} \in\{a, b\}} f\left(x_{1}, x_{2}, \ldots, x_{n}\right),
$$

and we use the same arguments for $x_{3}, \ldots, x_{n}$. Consequently, we obtain

$$
\max _{x \in[a, b]^{n}} f(x)=\max _{x \in\{a, b\}^{n}} f(x),
$$

where $x=\left(x_{1}, \ldots, x_{n}\right) \in \mathbb{R}^{n}$.

This completes our proof. 


\section{Applications of Theorem 2.1}

In this section we give the main results of our paper.

Application 3.1. Let $x_{1}, \ldots, x_{n} \in[a, b] \subset(0, \infty)$, where $a=\min _{i=1, n} x_{i}$ and $b=\max _{i=\overline{1, n}} x_{i}$. Then

$$
\frac{x_{1}+\cdots+x_{n}}{n}-\sqrt[n]{x_{1} \cdots x_{n}} \leq(L(a, b)-a)\left(\frac{L(a, b)}{L(a, L(a, b))}-1\right)
$$

where

$$
L(a, b)=\frac{b-a}{\log b-\log a},
$$

denotes the logarithmic mean of $a$ and $b$.

Proof. Consider the function $f:[a, b]^{n} \rightarrow \mathbb{R}$, given by the relation

$$
f\left(x_{1}, \ldots, x_{n}\right)=\frac{x_{1}+\cdots+x_{n}}{n}-\sqrt[n]{x_{1} \cdots x_{n}} .
$$

Take note that

$$
\frac{\partial^{2} f}{\partial x_{i}^{2}}=\frac{n-1}{n^{2}}\left(x_{1} \cdots x_{i-1} x_{i+1} \cdots x_{n}\right)^{\frac{1}{n}} x_{i}^{-\frac{2 n-1}{n}}
$$

for $i=\overline{1, n}$.

Since all partial derivatives of second order of $f$ are positive, by applying Theorem 2.1 we obtain

$$
\max _{x \in[a, b]^{n}} f(x)=\max _{x \in\{a, b\}^{n}} f(x) .
$$

We shall determine now $\max _{x \in\{a, b\}^{n}} f(x)$.

Without loss of generality, we may now assume that the maximum of $f$ is obtained at the point $\left(x_{1}, \ldots, x_{n}\right)$, where

$$
\begin{aligned}
& x_{1}=x_{2}=\cdots=x_{n-k}=a, \\
& x_{n-k+1}=x_{n-k+2}=\cdots=x_{n}=b .
\end{aligned}
$$

Hence, to determine $\max _{x \in\{a, b\}^{n}} f(x)$, it is enough to find the maximum of the expression

$$
E=\frac{(n-k) a+k b}{n}-\sqrt[n]{a^{n-k} b^{k}}=a+\frac{k}{n}(b-a)-a\left(\frac{a}{b}\right)^{\frac{k}{n}}
$$

for $k=\overline{1, n-1}$.

To this end, let $g:(0,1) \rightarrow \mathbb{R}$ be given by

$$
g(t)=a+t(b-a)-a\left(\frac{a}{b}\right)^{t} .
$$

The derivative of $g$ is as follows

$$
g^{\prime}(t)=b-a-a\left(\frac{b}{a}\right)^{t} \log \left(\frac{b}{a}\right) .
$$


Hence,

$$
g^{\prime}(t)=0 \Leftrightarrow\left(\frac{b}{a}\right)^{t}=\frac{L(a, b)}{a} \Leftrightarrow t^{\prime}:=t=\frac{\log (L(a, b))-\log a}{\log b-\log a} \in(0,1) .
$$

We deduce that $t^{\prime}$ is a global maximum point for $g$. Consequently,

$$
\max _{t \in(0,1)} g(t)=g\left(t^{\prime}\right) \text {. }
$$

Our claim is that

$$
g\left(t^{\prime}\right)=(L(a, b)-a)\left[\frac{L(a, b)}{L(a, L(a, b))}-1\right] .
$$

Indeed,

$$
\begin{aligned}
g\left(t^{\prime}\right) & =a+t^{\prime}(b-a)-a\left(\frac{b}{a}\right)^{t^{\prime}} \\
& =a+t^{\prime}(b-a)-a \frac{L(a, b)}{a} \\
& =a+\frac{b-a}{\log b-\log a} \cdot \frac{\log (L(a, b))-\log a}{L(a, b)-a}(L(a, b)-a)-L(a, b) \\
& =a+L(a, b) \frac{1}{L(a, L(a, b))}(L(a, b)-a)-L(a, b) \\
& =-(L(a, b)-a)+\frac{L(a, b)}{L(a, L(a, b))}(L(a, b)-a) \\
& =(L(a, b)-a)\left[\frac{L(a, b)}{L(a, L(a, b))}-1\right],
\end{aligned}
$$

and our claim is verified. This concludes the proof.

Application 3.2. Let $x_{1}, \ldots, x_{n} \in[a, b] \subset(0, \infty)$, where $a=\min _{i=1, n} x_{i}$ and $b=\max _{i=\overline{1, n}} x_{i}$. Then

$$
\frac{x_{1}+\cdots+x_{n}}{n}-\sqrt[n]{x_{1} \cdots x_{n}} \leq(\sqrt{b}-\sqrt{a})^{2} .
$$

Proof. Using similar arguments to those in the proof of Application 3.1, we consider the expression

$$
E=\frac{(n-k) a+k b}{n}-\sqrt[n]{a^{n-k} b^{k}}
$$

Note that,

$$
E \leq \frac{(n-k) a+k b}{n}-\frac{n}{\frac{n-k}{a}+\frac{k}{b}},
$$

due to the geometric and harmonic mean inequality, i.e.,

$$
\sqrt[n]{a^{n-k} b^{k}} \geq \frac{n}{\frac{n-k}{a}+\frac{k}{b}} .
$$


Thus, one obtains

$$
E \leq a+\frac{k}{n}(b-a)-\frac{a b}{b-\frac{k}{n}(b-a)} .
$$

On the other hand, we introduce the function $h:(0,1) \rightarrow \mathbb{R}$ as follows

$$
h(t)=1+t(b-a)-\frac{a b}{b-t(b-a)} .
$$

The derivative of the function $h$ is

$$
h^{\prime}(t)=b-a-\frac{a b}{(b-t(b-a))^{2}}(b-a) .
$$

Then we have

$$
h^{\prime}(t)=0 \Leftrightarrow t^{\prime}:=t=\frac{\sqrt{b}}{\sqrt{a}+\sqrt{b}} \in(0,1) .
$$

It follows that $t^{\prime}$ is a global maximum point for $h$ and therefore,

$$
h(t) \leq h\left(t^{\prime}\right)=(\sqrt{b}-\sqrt{a})^{2} .
$$

Consequently, we obtain

$$
\frac{x_{1}+\cdots+x_{n}}{n}-\sqrt[n]{x_{1} \cdots x_{n}} \leq(\sqrt{b}-\sqrt{a})^{2}
$$

and our proof is finished.

Now, we introduce the quantity

$$
P_{k}(a, b)=\sqrt[k]{\frac{b^{k+1}-a^{k+1}}{(k-1)(b-a)}}=\sqrt[k]{\frac{a^{k}+a^{k-1} b+\cdots+b^{k}}{k+1}},
$$

for $k \in \mathbb{N}, k \geq 2$ and $P_{1}(a, b)=\frac{a+b}{2}$.

One can easily see that $a<P_{k}(a, b)<b$, and thus $P_{k}(a, b)$ is a mean.

Application 3.3. Let $x_{1}, \ldots, x_{n} \in[a, b] \subset(0, \infty)$, where $a=\min _{i=1, n} x_{i}$ and $b=\max _{i=\overline{1, n}} x_{i}$. Then

$$
\sqrt[k]{\frac{x_{1}^{k}+\cdots+x_{n}^{k}}{n}}-\frac{x_{1}+\cdots+x_{n}}{n} \leq\left(P_{k-1}(a, b)-a\right)\left(1-\frac{P_{k-1}^{k-1}\left(a, P_{k-1}(a, b)\right)}{P_{k-1}^{k-1}(a, b)}\right) .
$$

Proof. Let $f:[a, b]^{n} \rightarrow \mathbb{R}$ be given by

$$
f\left(x_{1}, \ldots, x_{n}\right)=\sqrt[k]{\frac{x_{1}^{k}+\cdots+x_{n}^{k}}{n}}-\frac{x_{1}+\cdots+x_{n}}{n} .
$$

One can easily see that

$$
\frac{\partial^{2} f}{\partial x_{i}^{2}}=\frac{k-1}{n^{2}} x_{i}^{k-2}\left(\frac{x_{1}^{k}+\cdots+x_{n}^{k}}{n}\right)^{\frac{1}{k}-2} \frac{x_{1}^{k}+\cdots+x_{i-1}^{k}+x_{i+1}^{k}+\cdots+x_{n}^{k}}{n}>0,
$$

for all $i=\overline{1, n}$. 
We apply Theorem 2.1 and we obtain that

$$
\begin{aligned}
\max _{x \in[a,]^{n}} f(x) & =\max _{p \in\{1, \ldots, n-1\}} \sqrt[k]{\frac{(n-p) a^{k}+p b^{k}}{n}}-\frac{(n-a) p+p b}{n} \\
& =\max _{p \in\{1, \ldots, n-1\}}\left(\sqrt[k]{\frac{p}{n}\left(b^{k}-a^{k}\right)+a^{k}}-\frac{p}{n}(b-a)-a\right) .
\end{aligned}
$$

Consider, now, the function $g:(0,1) \rightarrow \mathbb{R}$ given by

$$
g(t)=\sqrt[k]{t\left(b^{k}-a^{k}\right)+a^{k}}-t(b-a)-a .
$$

We deduce that

$$
g^{\prime}(t)=\frac{b^{k}-a^{k}}{k\left(\sqrt[k]{t\left(b^{k}-a^{k}\right)+a^{k}}\right)^{k-1}}-(b-a) .
$$

Hence,

$$
g^{\prime}(t)=0 \Leftrightarrow \alpha:=t=\frac{P_{k-1}^{k}(a, b)-a^{k}}{b^{k}-a^{k}} \in(0,1) .
$$

Consequently, we have

$$
\max _{t \in(0,1)} g(t)=g(\alpha)
$$

On the other hand, we get

$$
\begin{aligned}
g(\alpha) & =P_{k-1}(a, b)-\frac{P_{k-1}^{k}(a, b)-a}{b^{k}-a^{k}}(b-a)-a \\
& =P_{k-1}(a, b)-a-\frac{k(b-a)}{b^{k}-a^{k}} \frac{P_{k-1}^{k}(a, b)-a^{k}}{k\left(P_{k-1}(a, b)-a\right)}\left(P_{k-1}(a, b)-a\right) \\
& =P_{k-1}(a, b)-a-\frac{1}{P_{k-1}^{k-1}}(a, b) P_{k-1}^{k-1}(a, P(a, b))\left(P_{k-1}(a, b)-a\right) \\
& =\left(P_{k-1}(a, b)-a\right)\left(1-\frac{P_{k-1}^{k-1}\left(a, P_{k-1}(a, b)\right)}{P_{k-1}^{k-1}(a, b)}\right)
\end{aligned}
$$

This concludes our proof.

Remark 3.1. Setting $n=2$ in Application 3.3 yields the following inequality

$$
\sqrt{\frac{x_{1}^{2}+\cdots+x_{n}^{2}}{n}}-\frac{x_{1}+\cdots+x_{n}}{n} \leq \frac{(b-a)^{2}}{4(a+b)} .
$$

In the latter, we shall state and prove Schweitzer's inequality.

Theorem 3.4 (Schweitzer). Let $x_{1}, \ldots, x_{n}, a, b>0$ such that $x_{i} \in[a, b]$ for all $i=\overline{1, n}$. Then,

$$
\begin{aligned}
& \left(x_{1}+\cdots+x_{n}\right)\left(\frac{1}{x_{1}}+\cdots+\frac{1}{x_{n}}\right) \leq \frac{(a+b)^{2}}{4 a b} n^{2}, \quad \text { for } n \text { even }, \\
& \left(x_{1}+\cdots+x_{n}\right)\left(\frac{1}{x_{1}}+\cdots+\frac{1}{x_{n}}\right) \leq \frac{(a+b)^{2}}{4 a b} n^{2}-\frac{(a-b)^{2}}{4 a b}, \text { for } n \text { odd } .
\end{aligned}
$$


Proof. Define $f:[a, b]^{n} \rightarrow \mathbb{R}$ as follows

$$
f\left(x_{1}, \ldots, x_{n}\right)=\left(x_{1}+\cdots+x_{n}\right)\left(\frac{1}{x_{1}}+\cdots+\frac{1}{x_{n}}\right) .
$$

Then, for all $i=\overline{1, n}$, we have

$$
\frac{\partial^{2} f}{\partial x_{i}^{2}}=\frac{2\left(x_{1}+\cdots+x_{i-1}+x_{i+1}+\cdots+x_{n}\right)}{x_{i}^{3}}>0 .
$$

Apply Theorem 2.1 and deduce that

$$
\max _{x \in[a, b]^{n}} f(x)=\max _{x \in\{a, b\}^{n}} f(x),
$$

where $x=\left(x_{1}, \ldots, x_{n}\right) \in \mathbb{R}^{n}$. Now, we determine $\max _{x \in\{a, b\}^{n}} f(x)$. Without loss of generality, we may assume that

$$
\begin{aligned}
& x_{1}=x_{2}=\cdots=x_{k}=a, \\
& x_{k+1}=x_{k+2}=\cdots=x_{n}=b .
\end{aligned}
$$

Hence,

$$
\max _{x \in\{a, b\}^{n}} f(x)=\max _{k \in\{1, \ldots, n\}}(k a+(n-k) b)\left(\frac{k}{a}+\frac{n-k}{b}\right) .
$$

We consider the function $g:(0, \infty) \rightarrow \mathbb{R}$, defined by

$$
g(k)=(k a+(n-k) b)\left(\frac{k}{a}+\frac{n-k}{b}\right) .
$$

A short computation yields

$$
g(k)=-\frac{(b-a)^{2}}{a b} k^{2}+\frac{n(b-a)^{2}}{a b} k+n^{2} .
$$

One can easily deduce that the maximum of $g$ is obtained when $x=\frac{n}{2}$. Moreover, the restriction of $g$ to the set $\{1, \ldots, n\}$ obtains its maximum value at $k=\frac{n}{2}$ if $n$ is even and $k=\frac{n-1}{2}$ or $k=\frac{n+1}{2}$ is $n$ is odd. On the other hand, take note that

$$
\begin{aligned}
& g\left(\frac{n}{2}\right)=\frac{(a+b)^{2}}{4 a b} n^{2}, \quad \text { for } n \text { even, } \\
& g\left(\frac{n-1}{2}\right)=g\left(\frac{n+1}{2}\right)=\frac{(a+b)^{2}}{4 a b} n^{2}-\frac{(b-a)^{2}}{4 a b}, \quad \text { for } n \text { odd. }
\end{aligned}
$$

This concludes our proof.

Now we focus on the statement and proof of Khanin's inequality. 
Theorem 3.5 (Khanin). Let $x_{1}, \ldots, x_{n}, a, b \in \mathbb{R}$ such that $x_{i} \in[a, b]$ for all $i=\overline{1, n}$. Then

$$
\begin{aligned}
& \frac{x_{1}^{2}+\cdots+x_{n}^{2}}{n}-\left(\frac{x_{1}+\cdots+x_{n}}{n}\right)^{2} \leq \frac{(b-a)^{2}}{4}, \quad \text { for } n \text { even, } \\
& \frac{x_{1}^{2}+\cdots+x_{n}^{2}}{n}-\left(\frac{x_{1}+\cdots+x_{n}}{n}\right)^{2} \leq\left(\frac{b-a}{4}\right)^{2}-\frac{(b-a)^{2}}{4 n^{2}}, \quad \text { for } n \text { odd. }
\end{aligned}
$$

Proof. Let $f:[a, b]^{n} \rightarrow \mathbb{R}$, given by

$$
f\left(x_{1}, \ldots, x_{n}\right)=\frac{x_{1}^{2}+\cdots+x_{n}^{2}}{n}-\left(\frac{x_{1}+\cdots+x_{n}}{n}\right)^{2} .
$$

Note that

$$
\frac{\partial^{2} f}{\partial x_{i}^{2}}=\frac{2(n-1)}{n^{2}}>0 .
$$

Now, apply Theorem 2.1 and deduce that

$$
\frac{x_{1}^{2}+\cdots+x_{n}^{2}}{n}-\left(\frac{x_{1}+\cdots+x_{n}}{n}\right)^{2} \leq \frac{k a^{2}+(n-k) b^{2}}{n}-\left(\frac{k a+(n-k) b}{n}\right)^{2} .
$$

Further, we consider the function $g:(0, \infty) \rightarrow \mathbb{R}$, given by

$$
g(k)=\frac{k a^{2}+(n-k) b^{2}}{n}-\left(\frac{k a+(n-k) b}{n}\right)^{2} .
$$

It can be easily seen that the restriction of $g$, i.e., $g:\{1,2, \ldots, n\} \rightarrow \mathbb{R}$ obtains its maximum value when $k=\frac{n}{2}$ if $n$ is even and when $k=\frac{n-1}{2}$ or $k=\frac{n+1}{2}$ if $n$ is odd. Taking note that

$$
\begin{aligned}
g\left(\frac{n}{2}\right) & =\frac{(b-a)^{2}}{4}, \quad \text { for } n \text { even, } \\
g\left(\frac{n-1}{2}\right) & =g\left(\frac{n+1}{2}\right)=\left(\frac{b-a}{4}\right)^{2}-\frac{(b-a)^{2}}{4 n^{2}}, \quad \text { for } n \text { odd },
\end{aligned}
$$

we obtain relations (3.1).

This completes our proof.

\section{REFERENCES}

[1] E. F. Beckenbach and R. Bellman, Inequalities, Springer-Verlag, Berlin, 1961.

[2] W. W. Breckner and T. Trif, Convex Functions and Related Functional Equations, Presa Universitară Clujeană, 2008.

[3] P. S. Bullen, A Dictionary of Inequalities, Kluwer Academic Publishers, Dordrecht, 2003.

[4] P. Cerrone and S. S. Dragomir, Mathematical Inequalities, A Perspective, CRC Press, Boca Raton, London, New York, 2011.

[5] I. Maruşciac, Programare Geometrică şi Aplicaţii, Dacia, Cluj-Napoca, 1978.

[6] D. S. Mitrinović, J. E. Pećarić and A. M. Fink, Classical and New Inequalities in Analysis, Kluwer Academic Publishers, Dordrecht, 1993. 
[7] C. Niculescu and L. E. Persson, Convex Functions and their Applications, Springer-Verlag, New York, 2006.

[8] A. Roberts and D. Varberg, Convex Functions, Academic Press, New York, 1973.

${ }^{1}$ Faculty of Mathematics and Computer Science, BABEŞ-Bolyai University,

1, Kogălniceanu Street, 400084 Cluj-Napoca, Romania

Email address: florin.albisoru@gmail.com

${ }^{2}$ Emanuil Gojdu High School,

1 Bis, Al. Vlahuţă Street, 331067 Hunedoara, Romania

Email address: maricu_stroe@yahoo.com 